\title{
Case Studies in Adult Intensive Care Medicine
}

\author{
Daniele Bryden, Andrew Temple (Editors). Cambridge University Press, United \\ Kingdom, 2017. \$75, 372 pages, ISBN 978-1-107-42337-4
}

\author{
Mika Hamilton, MBChB
}

Received: 24 November 2017/Accepted: 27 November 2017/Published online: 18 December 2017

(c) Canadian Anesthesiologists' Society 2017

Editors Bryden and Temple call on the experience of multiple clinicians in the field of intensive care medicine (ICM) to bring us a series of case studies, each chosen to highlight, discuss, and educate clinicians in particular areas of importance to this specialty. Although targeted at qualifying examination candidates, this book is not intended to be a definitive text on all aspects of ICM. Rather, these cases are designed to aid in preparation for critical care licensing examinations - particularly, the UK Faculty of Intensive Care Medicine examination (to become a Fellow of the Faculty of Intensive Care Medicine) and the European Society of Intensive Care Medicine examination (for a European Diploma in Intensive Care Medicine) - by acting as a basis for independent study and providing material for group tutorials. The case studies also provide details of clinical scenarios that are of interest to all health care professionals looking after critically ill patients.

The book is composed of 45 short chapters, each composed of an introduction to a topic, description of a clinical case, discussion around areas of importance, a conclusion, and a final summary of key learning points. Pertinent references are provided, so although each chapter can be read within a short period of time, there is direction toward, and opportunity for, further learning.

The case studies presented are described as "real-life" situations and quickly capture the reader's interest. We are introduced to the patient, witness the patient's presentation with, or progression to, critical illness, observe the clinician's actions, and conclude with the patient's

M. Hamilton, MBChB ( $\square)$

James Cook University Hospital, Middlesbrough, UK

e-mail: mikahamilton@doctors.org.uk outcome. Indeed, the case studies in each chapter are a good introduction to the subsequent discussion. They allow the reader to contemplate different aspects of the case, consider their prior knowledge of the evidence for the interventions described, think "what would I do/have done if I was the clinician looking after this patient," and reflect on the case - all before moving on to the discussion.

The realistic nature of the case studies is refreshing. Some interventions described are acknowledged as not evidence-based, but their use is explained as "a result of local practice." These deviations from evidence-based medicine prompt readers to reflect on their own practice while highlighting the importance of critical appraisal of local dogma. The use of real-life case studies also reminds the reader that ICM requires more than memorizing a syllabus - that it is the individual patient who is at the core of everything we do. This concept is beautifully demonstrated in the photographs included in a case study of a patient who had suffered severe burns. The authors presented images of the young woman on the operating table undergoing burn wound debridement and grafting and, subsequently, at a follow-up clinic, smiling.

There is good use made of tables and diagrams within the text, as well as a number of clinical, radiological, and histological images. Although these tools help break up the text and simplify or highlight certain information, they cause an occasional chapter to be fairly lengthy. Most chapters remain reasonably short, however, allowing the reader an opportunity to digest their dense nature quickly.

The information in this book is, for the most part, accurate and as up to date as possible. The authors clearly acknowledge situations where relevant evidence is currently being studied, has potential to change, or is contentious. Occasionally, however, the facts provided are 
erroneous. For example, in the chapter discussing necrotizing soft tissue infections, the information delivered regarding the salience of predisposing factors in various types of necrotizing fasciitis contradicts itself between the introduction and the conclusion. There are also a few spelling and grammatical errors in the book, and on occasion the graphics within accompanying tables and diagrams do not quite line up properly. Lastly, the book synopsis quotes "48 case studies" when there appears to be only 45. Although these errors are noticeable, they are easily rectifiable in a future edition.

This book is a good collection of relevant and interesting case studies, pragmatic discussions, and appropriate references for further education. The cases described may be familiar or unfamiliar to the reader, allowing both learning of new material and consolidation of previously reviewed topics. Although not designed to be an exhaustive text on all aspects of ICM, the authors have fulfilled their objective of providing an excellent source of reading material for examination preparation, group discussion, and individual learning. Despite some minor errors, which can be easily corrected in a future edition, I enjoyed reading this book and found it a useful reference. I would recommend it not only to individuals preparing for ICM examinations, but also as an educational source for all professionals looking after critically ill patients, and as a refresher for experienced intensive care physicians.

Conflicts of interest None declared.

Editorial responsibility This submission was handled by Dr. Hilary P. Grocott, Editor-in-Chief, Canadian Journal of Anesthesia. 